OPEN ACCESS

Edited by:

Wu Yuan,

The Chinese University of Hong

Kong, China

Reviewed by:

Smriti Murali Krishna,

James Cook University, Australia

Jes Lindholt,

Odense University Hospital, Denmark

${ }^{*}$ Correspondence:

Zhenjie Liu

lawson4001@zju.edu.cn

tThese authors have contributed equally to this work

Specialty section:

This article was submitted to

Translational Medicine,

a section of the journal

Frontiers in Medicine

Received: 09 February 2021 Accepted: 03 June 2021 Published: 07 July 2021

Citation:

Yin L, Zhang K, Sun Y and Liu Z (2021)

Nanoparticle-Assisted Diagnosis and

Treatment for Abdominal Aortic

Aneurysm. Front. Med. 8:665846.

doi: 10.3389/fmed.2021.665846

\section{Nanoparticle-Assisted Diagnosis and Treatment for Abdominal Aortic Aneurysm}

\author{
Li Yin $^{1 \dagger}$, Kaijie Zhang ${ }^{2 \dagger}$, Yuting Sun ${ }^{3}$ and Zhenjie Liu ${ }^{1 *}$ \\ ${ }^{1}$ Department of Vascular Surgery, The Second Affiliated Hospital of Zhejiang University School of Medicine, Hangzhou, \\ China, ${ }^{2}$ Department of Cardiology, Sir Run Run Shaw Hospital of Zhejiang University School of Medicine, Hangzhou, China, \\ ${ }^{3}$ Department of Orthopaedic Surgery, Sir Run Run Shaw Hospital of Zhejiang University School of Medicine, Hangzhou, China
}

An abdominal aortic aneurysm (AAA) is a localized dilatation of the aorta related to the regional weakening of the wall structure, resulting in substantial morbidity and mortality with the aortic ruptures as complications. Ruptured AAA is a dramatic catastrophe, and aortic emergencies constitute one of the leading causes of acute death in older adults. AAA management has been centered on surgical repair of larger aneurysms to mitigate the risks of rupture, and curative early diagnosis and effective pharmacological treatments for this condition are still lacking. Nanoscience provided a possibility of more targeted imaging and drug delivery system. Multifunctional nanoparticles (NPs) may be modified with ligands or biomembranes to target agents' delivery to the lesion site, thus reducing systemic toxicity. Furthermore, NPs can improve drug solubility, circulation time, bioavailability, and efficacy after systemic administration. The varied judiciously engineered nano-biomaterials can exist stably in the blood vessels for a long time without being taken up by cells. Here, in this review, we focused on the NP application in the imaging and treatment of AAA. We hope to make an overview of NP-assisted diagnoses and therapy in AAA and discussed the potential of NP-assisted treatment.

Keywords: nanoparticles, abdominal aortic aneurysm, imaging, treatment, diagnosis

\section{INTRODUCTION}

An abdominal aortic aneurysm (AAA) is a localized dilatation of the aorta related to the regional weakening of the wall structure, resulting in substantial morbidity and mortality with the aortic ruptures as complications. The disease's prevalence depends on the population studied, with reported prevalence varying between 1.4 and $12.4 \%$ (1). AAAs are generally asymptomatic and are usually diagnosed by screening or as an incidental finding. The natural history of the disease is that of slow progression and ultimate rupture (2). Ruptured AAA is a dramatic catastrophe, and aortic emergencies constitute one of the leading causes of acute death in older adults (2). Multiple trials have shown no benefit of repair of AAA at sizes $<55 \mathrm{~mm}$ in diameter, and consequently, current guidelines advise watchful waiting for aneurysms $<55 \mathrm{~mm}$ and preventive repair once the AAA grows $>55 \mathrm{~mm}(3,4)$, possibly with a slightly lower intervention threshold for repair in women. AAA management has been centered for decades on surgical repair of larger aneurysms to mitigate the risks of rupture, and remarkable progress has been achieved in both open and endovascular surgery, but curative early diagnosis and effective pharmacological treatments for this condition are still lacking (5). Screening for AAA needs more imaging techniques. Many drugs such as doxycycline and rapamycin demonstrated their efficacies in animal models. However, they showed 
low efficacy in clinical trials, potentially because of the lack of targeted drug delivery. A significant challenge is delivering effective dosage to local lesion sites without any adverse effects $(6,7)$.

Nanoscience provided a possibility of more targeted imaging and drug delivery system. Nanoparticles (NPs) are particles in the nanometer range $(1-100 \mathrm{~nm})$, which are more than 100 times smaller than human cells, and have biological activity and fluidity in both the internal and external blood vessel systems (8). Multifunctional NPs have recently garnered considerable attention for applications that may revolutionize imaging and drug delivery. NPs can be modified with ligands or biomembranes to target therapeutic agents' targeted delivery to the lesion site, thus reducing systemic toxicity. Furthermore, NPs can improve drug solubility, circulation time, bioavailability, and efficacy after systemic administration. Judiciously engineered NPs can enable therapy and diagnosis simultaneously (912). Nanoscience provides a possibility that NPs can bind specific areas like degraded elastic lamina, inflammation cells, or peptides. The varied nano-biomaterials can exist stably in the blood vessels for a long time without being taken up by cells. Several reports exist with a focus on the application of NPs for enhanced imaging of aneurysmal lesions in live animals as well as in patients. It is noteworthy, however, despite relative scarcity, that recent reports have shown exciting progress in developing NP-based approaches to treat AAA via targeted delivery.

Here in this review, we focused on the NP application in AAA. We summarized the NP-assisted imaging and treatment of AAA to overview the value of NP in AAA diagnosis and treatment and discuss the potential use in AAA.

\section{NANOPARTICLES IN ABDOMINAL AORTIC ANEURYSM IMAGING}

Most AAAs are asymptomatic until severe complications such as rupture occur, leading to patients' sudden death (13). On the other hand, the progression rate of AAA is highly variable among different individuals. Therefore, it is vital to develop novel imaging tools and techniques for improving the sensibility and accuracy to diagnose AAA at an early stage $(10,14)$. Compared with conventional contrast agents, NPs have prolonged blood circulation time, controlled biological clearance pathways, and specific molecular targeting capabilities $(15,16)$. Due to their modifiability and biocompatibility, NPs have been reported in several studies as an agent for AAA imaging $(9,17,18)$. At present, most of NPs used as contrast agents for AAA imaging are not be modified by specific targeted ligands. However, due to the infiltration of inflammatory cells, especially phagocytes that can uptake NPs, these NPs can still be detected in AAA lesion

\footnotetext{
Abbreviations: AAA, abdominal aortic aneurysm; CT, computed tomography; ECM, extracellular matrix; EVAR, endovascular aneurysm repair; FDG, fluorodeoxyglucose; HA, hyaluronic acid; MMP, matrix metalloproteinase; MRI, magnetic resonance imaging; NP, nanoparticle; PET, positron emission tomography; ROS, reactive oxygen species; SPIO, superparamagnetic iron oxide; USPIO, ultrasmall superparamagnetic particles of iron oxide; VSMC, vascular smooth muscle cell.
}

by several imaging techniques. Compared with that, previous studies have shown that NPs that are surface-modified are prone to highly accumulate at AAA lesion and show greater imaging intensity. An overview of application of NPs in several imaging techniques may improve the understanding of NP-assisted AAA imaging (see Table 1).

\section{Nanoparticles in Computed Tomography}

CT provides a non-invasive imaging capability with higher spatial and temporal resolution but lowers sensitivity as compared with other clinical imaging methods (PET and MRI). There have been studies exploring the application of CT in the diagnosis of AAA. For example, CT signal heterogeneity measurements in small AAAs have been revealed as a useful risk stratification tool to identify aneurysms with a high risk of significant expansion (33). Gold NPs (AuNPs) have attracted much attention as an X-ray contrast agent in recent years due to their high X-ray attenuation, non-toxicity, easy synthesis, and surface functionalization (19). Compared with barium sulfate and iodine, gold shows high $\mathrm{X}$-ray attenuation coefficient, especially in the energy level for clinical CT. AuNPs exhibit longer vascular residence time than iodide molecules because of their higher molecular weight, which may increase the available imaging window (34). Au et al. (35) reported that AuNPs could provide clear and durable contrast enhancement of the vasculature even at $24 \mathrm{~h}$ (20). Studies have shown that AuNPs could be used to effectively label several cell types, including stem cells and immune cells, without damaging their activities. This could be used as a novel method for in vivo tracking of therapeutic cells (21). Using an angiotensin II (ANG II)-induced AAA mouse model, Wang et al. (36) revealed in vivo targeting of AuNPs conjugated with elastin (EL-AuNPs) of damaged aortic elastin in AAA lesions, and a correlation between micro-CT-based signal intensities and burst pressures was found. Lane et al. also used EL-AuNPs as a diagnostic tool for evaluating unruptured AAAs. Electron dense EL-AuNPs were visualized by micro-CT, and the corresponding gold-to-tissue volume ratios quantified. The gold-to-tissue volume ratios correlated strongly with the concentration of infused porcine pancreatic elastase and therefore the degree of elastin damage (37). These findings all indicate that AuNPs could become useful contrast agents for AAA CT imaging.

\section{Nanoparticles in Positron Emission Tomography}

PET is a non-invasive technique that is also widely used in clinical practice. Through radiolabeled tracers in target tissues, PET enables visualization of several biochemical processes effectively at the cellular and molecular levels. Since AAA pathology is associated with inflammatory cell infiltrate and enzymatic degradation of the vessel wall, there have been numerous PET imaging reports in AAA patients. PET imaging revealed increased fluorodeoxyglucose $\left({ }^{18} \mathrm{~F}-\mathrm{FDG}\right)$ uptake in patients with AAA, suggesting a possible association between increased ${ }^{18} \mathrm{~F}$ FDG uptake and AAA expansion and rupture (38-41). PET combined with CT rays scan (PET-CT) has also been revealed as a promising technique to identify the aneurysm wall (4244). However, a study reported that the chronic inflammation 
TABLE 1 | Nanoparticles in AAA imaging.

\begin{tabular}{|c|c|c|c|c|c|}
\hline Nanoparticles & Ligands & Targets & Imaging technique & Models & Detailed application \\
\hline AuNPs & $\begin{array}{l}\text { Methoxy-polyethylene } \\
\text { glycol-thiol chains }\end{array}$ & NA & CT & Murine models & $\begin{array}{l}\text { Provide clear and durable contrast } \\
\text { enhancement of the vasculature even at } \\
24 \mathrm{~h} \text { (19). }\end{array}$ \\
\hline EL-AuNPs & An elastin antibody & Elastin & CT & $\begin{array}{l}\text { Ang II infusion in LDLr }{ }^{-/-} \\
\text {mice }\end{array}$ & $\begin{array}{l}\text { In vivo targeting to damaged aortic elastin } \\
\text { in mice with AAA (20). }\end{array}$ \\
\hline EL-AuNPs & $\begin{array}{l}\text { An antibody against } \\
\text { fragmented elastin }\end{array}$ & Fragmented elastin & CT & $\begin{array}{l}\text { Porcine pancreatic } \\
\text { elastase-induced AAA mice }\end{array}$ & $\begin{array}{l}\text { Non-destructive estimation of mechanical } \\
\text { and geometric features of AAAs when } \\
\text { combined with CT (21). }\end{array}$ \\
\hline $\begin{array}{l}\text { Monocyte/ } \\
\text { macrophage- } \\
\text { targeted } \\
\text { NPs }\end{array}$ & fluorine-18 [(18)F] & Monocyte/macrophage & PET-CT & $\begin{array}{l}\text { ANG II infusion in apoE }{ }^{-/-} \\
\text {mice }\end{array}$ & $\begin{array}{l}\text { Quantitation of macrophage content in a } \\
\text { mouse AAA model (22). }\end{array}$ \\
\hline Iron oxide NPs & NA & $\begin{array}{l}\text { Vascular smooth } \\
\text { muscle cells (VSMCs) }\end{array}$ & MRI & $\begin{array}{l}\text { Male Fisher rats implanted } \\
\text { with ECM tubes }\end{array}$ & $\begin{array}{l}\text { Evaluating whether magnetic cell labeling } \\
\text { can be used to non-invasively assess the } \\
\text { technical success of endovascular cell } \\
\text { therapy for AAAs (23). }\end{array}$ \\
\hline $\begin{array}{l}\text { Human ferritin NPs } \\
\text { (HFn) }\end{array}$ & Arg-Gly-Asp (RGD) & $\begin{array}{l}\text { Vascular inflammation } \\
\text { and angiogenesis }\end{array}$ & MRI & $\begin{array}{l}\text { Ang } \| \text { infusion in apoE }{ }^{-/-} \\
\text {mice }\end{array}$ & $\begin{array}{l}\text { Enhances vascular molecular imaging by } \\
\text { targeting both vascular inflammation and } \\
\text { angiogenesis (24). }\end{array}$ \\
\hline USPIO & NA & NA & MRI & Asymptomatic AAA patients & $\begin{array}{l}\text { To distinguish those patients with more } \\
\text { rapidly progressive AAA expansion (25). }\end{array}$ \\
\hline $\begin{array}{l}\text { Paramagnetic/ } \\
\text { fluorescent } \\
\text { micellar NPs }\end{array}$ & $\begin{array}{l}\text { Collagen-binding } \\
\text { protein (CNA-35) }\end{array}$ & Collagen & MRI & $\begin{array}{l}\text { Ang II infusion combined } \\
\text { with TGF- } \beta \text { neutralization } \\
\text { mouse model }\end{array}$ & $\begin{array}{l}\text { The presence of collagen was visualized } \\
\text { by nanoparticle-enhanced MRI in AAA } \\
\text { (26). }\end{array}$ \\
\hline USPIO & NA & NA & MRI & Patients with AAA & $\begin{array}{l}\text { Detection of vascular inflammation to } \\
\text { further assess AAA rupture risk (27). }\end{array}$ \\
\hline SPIO & NA & Macrophages & MRI & $\begin{array}{l}\text { Ang II infusion in apoE }{ }^{-/-} \\
\text {mice }\end{array}$ & $\begin{array}{l}\text { Detection of macrophage activities in an } \\
\text { ANG II-induced early-stage AAA model } \\
\text { (28). }\end{array}$ \\
\hline Nanoparticles & Ligands & Targets & Imaging technique & Models & Detailed application \\
\hline SPIO & NA & NA & MRI & $\begin{array}{l}\text { AAA patients within two } \\
\text { weeks after EVAR }\end{array}$ & $\begin{array}{l}\text { Detection of endoleaks in patients with } \\
\text { AAA after EVAR (29). }\end{array}$ \\
\hline Iron oxide NPs & NA & Macrophages & $\begin{array}{l}\text { bioluminescence (BLI) } \\
\text { and MRI }\end{array}$ & $\begin{array}{l}\text { Porcine pancreatic } \\
\text { elastase-induced AAA mice }\end{array}$ & $\begin{array}{l}\text { Exploring macrophage homing and } \\
\text { accumulation in experimental AAA disease } \\
\text { (30). }\end{array}$ \\
\hline $\begin{array}{l}\text { Core/shell Fe/iron } \\
\text { oxide NPs }\end{array}$ & MMP peptide substrate & MMP & MRI & $\begin{array}{l}\text { An Ang II-induced AAA } \\
\text { model }\end{array}$ & $\begin{array}{l}\text { Detection of MMP activity within the AAA } \\
\text { wall, thus representing a potential } \\
\text { non-invasive method to predict the rupture } \\
\text { risk of AAA (31). }\end{array}$ \\
\hline Magnetic NPs & NA & NA & $\begin{array}{l}\text { Magnetic particle } \\
\text { imaging (MPI) }\end{array}$ & $\begin{array}{l}\text { Ang II-induced ApoE } E^{-/-} \\
\text {mice }\end{array}$ & $\begin{array}{l}\text { Monitoring inflammatory progression in } \\
\text { AAA in an experimental setting (32). }\end{array}$ \\
\hline
\end{tabular}

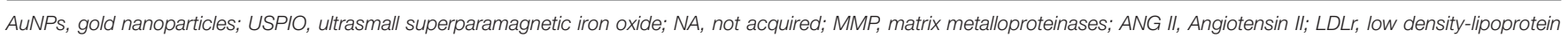
receptor; apoE, apolipoprotein E; EVAR, endovascular aneurysm repair.

observed on the asymptomatic AAA wall was not metabolically sufficient to cause the increased glucose metabolism detected by FDG-PET (45). In patients with sub-renal AAA, the metabolic activity level of ${ }^{18}$ F-FDG PET-CT was not associated with the aortic size $(46,47)$. Therefore, the association between ${ }^{18} \mathrm{~F}$ FDG uptake and inflammation in AAA is still controversial. It is necessary to develop novel PET tracers to investigate the pathogenesis of AAA further.

Several studies reported the application of NPs as an emerging molecular imaging tool in PET imaging $(22,48)$. However, few studies have investigated the application of NPs in AAA PET imaging. Nahrendorf et al. developed ${ }^{18}$ F-FDG-labeled NPs for PET-CT imaging. These macrophage-targeted NPs labeled with fluorine-18 were used to quantify macrophages in ANG II-induced apoE ${ }^{-/-}$mouse model of AAA. The in vivo PET signal was significantly higher in AAA compared with wild-type aorta (49). In general, our knowledge of the application of NPs in AAA PET imaging is limited, and more studies are required to explore the role of NPs in AAA PET imaging thoroughly.

\section{Nanoparticles in Magnetic Resonance Imaging}

MRI has been widely used in clinical practice due to its noninvasive characteristic. Based on nuclear magnetic resonance 
(NMR), MRI can obtain three-dimensional tomographical information with high temporal-spatial resolution in whole tissue samples. The protons' alignment was generated in the body by a powerful magnetic field and then excited by a radiofrequency pulse ( $\mathrm{RF}$ pulse), causing proton resonance. The energy is released, and the proton spins back to the equilibrium state after being removed from the RF pulse, which can be detected by MRI sensors without involving harmful radioactivity (23). T1weighted images can be generated from T1 (longitudinal) signals, while T2-weighted images (T2WIs) can be generated from T2 (transverse) signals (50).

There have been several studies exploring the application of NPs in MRI for AAA. Deux et al. developed iron oxide NPs for labeling vascular smooth muscle cells (VSMCs), which were used to identify further magnetic cell labeling's ability in assessing the success of endovascular cell therapy for AAA. T2*weighted gradient-echo images showed areas of hypointense signal within the aortic wall immediately and up to 1 month after cell therapy, indicating that MRI with magnetic cell labeling can be used to document endovascular cell delivery (51). Kitagawa et al. developed Arg-Gly-Asp (RGD)-conjugated human ferritin (HFn) iron oxide NPs, which were then proved to enhance in vivo MRI by targeting both vascular inflammation and angiogenesis. Histological analysis showed colocalization of RGD-HFn- $\mathrm{Fe}_{3} \mathrm{O}_{4}$ to both macrophages infiltrating the vessel wall and adventitial endothelial cells, with greater uptake compared with that of nontargeted $\mathrm{HFn}-\mathrm{Fe}_{3} \mathrm{O}_{4}$ in AAA lesions. This provides a promising translatable MRI approach to detect high-risk atherosclerotic and aneurysmal vascular diseases (26).

Since collagen is a significant AAA component, NPs targeting collagen could be beneficial in AAA imaging (52). In a previous study, paramagnetic/fluorescent micellar NPs functionalized with a collagen-binding protein (CNA-35) were intravenously administered into $\mathrm{C} 57 \mathrm{BL} / 6$ mice with AAA. A significant higher MR signal enhancement was observed following the injection of CAN-35 in the aneurysmal wall compared with nonspecific micelles. Confocal microscopy demonstrated the precise colocalization of CNA-35 micelles with collagen-I. This study indicated that the presence of collagen could be visualized by NP-enhanced MRI (53).

Macrophage infiltration plays an essential role in AAA pathology $(25,52,54)$. Due to its characteristic of accumulating macrophages, ultrasmall superparamagnetic particles of iron oxide (USPIO) have been widely used for effectively quantifying the related inflammatory processes. Several studies have been conducted to assess whether cellular inflammation areas correlated with the rate of AAA expansion. In these studies, patients with AAA were imaged using MRI before and after administration of USPIO $(27,28,55,56)$. These studies' results emphasized that accumulation of USPIO in AAA could distinguish patients with more rapidly progressive AAA expansion. According to a study comparing combined ${ }^{18} \mathrm{~F}-\mathrm{FDG}$ PET-CT and USPIO-enhanced MRI, both ${ }^{18}$ F-FDG PET-CT and USPIO-MRI uptake identify vascular inflammation associated with AAA (29). Superparamagnetic iron oxide (SPIO) particles can be phagocytosed by macrophages after injection, which can be visualized using MRI as areas of signal loss caused by MRI susceptibility on T2WI. SPIO particles were used in a previous study as a marker to detect macrophage activities in an ANG II-induced early-stage AAA model, and the results indicated that SPIO could be used in imaging inflammation associated with AAA (31). SPIO-enhanced dynamic MRI was used as a potential alternative to contrast-enhanced CT for detecting endoleaks after endovascular aneurysm repair (EVAR) in a study. Eight of 11 endoleaks were detected by contrast-enhanced CT (8/11:73\%), while 10 endoleaks were detected by SPIOenhanced MRI (10/11:91\%), indicating that SPIO-enhanced MRI could detect more endoleaks than contrast-enhanced CT (57).

Matrix metalloproteinase (MMP) activation contributes to AAA growth and rupture. Yao et al. conducted a study to evaluate the ability of a novel activatable MRI nanoprobe to target MMPs in an ANG II-induced AAA model (58). This nanoprobe is composed of a hydrophilic polyethylene glycol (PEG) coating layer immobilized on the external surface of hydrophobic core/shell Fe/iron oxide NPs (Fe/IONPs); between them, there is a grafted MMP peptide substrate. In the mice injected with MMP-Fe/IONPs, a clear signal loss on T2WI sequences in the aneurysmal region was observed. The results indicated that MRI combined with nanoprobe allows detecting MMP activity within the AAA wall, thus representing a potential non-invasive method to predict the rupture risk of AAA.

\section{Nanoparticles in Other Imaging Techniques}

Due to the advantages mentioned above, NPs were also applied in many other techniques for AAA imaging (24). Integrin $\alpha \mathrm{V} \beta 3$ is expressed by monocytes and macrophages, which plays an essential role in vascular diseases such as AAA. Razavian et al. used an $\alpha \mathrm{V}$ integrin-specific tracer $\left({ }^{99 \mathrm{~m}} \mathrm{Tc}-\mathrm{NC} 100692\right)$ to investigate integrin-targeted imaging for detecting vessel wall inflammation (30). Arg-Gly-Asp (RGD)-conjugated HFn NPs were developed to evaluate inflammation and angiogenesis in AAA disease by near-infrared fluorescence imaging. RGD-HFn showed significantly higher signal than HFn in AAAs, and histology showed that RGD-HFn colocalized with macrophages and neoangiogenesis in AAA lesions (59). Sinha et al. developed NPs as imaging agents targeting degraded elastic lamina, a consistent pathological feature in vascular diseases (9). Based on murine AAA models, Miyama et al. developed a novel combined imaging approach with bioluminescence and MRI to study macrophage homing and accumulation in AAA, which provide novel insight into the investigation on AAA biology (32). Magnetic particle imaging (MPI) is an innovative imaging method, which can detect magnetic NPs (MNPs) with high sensitivity (60). Therefore, MPI is suitable as surrogate marker for molecular targeting of vascular inflammation. The quantitative ability for mapping MNPs establishes MPI as a promising tool for monitoring inflammatory progression in AAA as assessed by a recent study (61). In general, NPs will be applied in more and more novel techniques for AAA imaging as our understanding of NPs continues to advance. 


\section{NANOPARTICLES IN ABDOMINAL AORTIC ANEURYSM TREATMENT}

Surgery and endovascular therapy are still the primary central management of AAA. There is no therapeutics available in the clinic for AAA treatment. Many drugs demonstrated efficacy and safety in animal models but performed severely in the clinic. A significant challenge is delivering effective dosage to local lesion sites without any adverse effects $(6,7)$. NP-assisted AAA treatment is based on the NP-assisted delivery approach, similar to the NP-assisted imaging technique (see Figure 1). Researchers developed NP-assisted drug delivery performance of nanocarriers for AAA treatment targeting elastase laminae and AAA lesions (see Table 2).

\section{Nanoparticles Targeting the Elastic Laminae}

Elastic laminae in the tunica media are formed by elastin. The elastin degradation is a pathological characteristic of aortic aneurysms. Thus, elastin is used as a target for NP (9, 62, 66-74). Sinha et al. developed an elastin antibody (EL)-modified PEGylated poly(D,L-lactide) (PLA) nanoparticles (EL-PEG-PLA NPs) binding in the elastic laminae in elastase perfusion model and $\mathrm{CaCl}_{2}$ model (9). They observed that ELPEG-PLA NPs were capable of AAA targeting after systemic administration and were observed in the aortic wall's medial layer. Then they used PLA-NPs to load 1,1-dioctadecyl-3,3,3,3tetramethylindotricarbocyanine iodide (DIR) dyes and surface maleimide groups conjugated to thiolate elastin/IgG antibodies, targeted for degraded elastin layer. DIR dyes were indelibly associated with particles to facilitate visualization and tracking of NPs (9). They found that NPs are sensitive to elastic injury and precise spatial accumulation even under highshear hemodynamic conditions. Thus, EL-PEG-PLA NP was a predicting approach for AAA treatment when binding with certain drugs since these PLA NPs could locate diseased vascular lesson and avoid normal areas.

MMP inhibitors, which attenuate extracellular matrix (ECM) degradation, represent a promising drug class for AAA treatment. However, thus far, none of them has reached clinic success. The main limiting factors are low efficacy at low dosages but high toxicity at high dosages, quick activity loss in blood circulation, and low water solubility $(7,69)$. Researchers developed NP-assisted drug delivery systems that drugs can accumulate at AAA lesions depending on the limiting factors. Batimastat (BB-94) was a small-molecule inhibitor of MMPs initially developed for cancer therapy (75). The Sinha group first tested it in AAA treatment using EL-PEG-PLA NPs. Targeted delivery of BB-94-loaded EL-PEG-PLA NPs showed significantly improved efficacies in suppressing MMP activity and reducing elastin degradation, calcification, and aneurysm growth, compared with the control of free BB-94 without using EL-PEGPLA NPs in the $\mathrm{CaCl}_{2}$-induced rat aortic injury model. This improvement may be explained by increased drug solubility and bioavailability yet decreased adverse effects following systemic administration using EL-PEG-PLA NPs (69).
Doxycycline is a tetracycline derivative that has also been shown to inhibit the transcription process of MMP genes. Although, doxycycline has been widely studied as a drug for AAA treatment, it showed no clear clinical benefits in AAA patients (76). Recent studies found that with local therapy via mini-osmotic pumps or peri-aortic foams, the doxycycline dosage sufficient to inhibit MMP activities in vivo could be lowered $(63,64)$. Sylvester et al. (67) reported the application of cationic-functionalized poly(lactic-co-glycolic acid) (PLGA) NPs for elastin-targeted delivery of doxycycline to AAA sites (67). Furthermore, they recently reported several new nanoplatforms for AAA treatment. The doxycycline-loaded PEG-PLGA NPs were synthesized via a double-emulsion solvent evaporation technique using a di-dodecyl dimethyl ammonium bromide (DMAB), a cationic surfactant, followed by PEGylation to prolong the blood circulation time and increase the water solubility (77). Other researchers used another NP platform, the PLGA NPs, to co-encapsulate doxycycline and SPION to inhibit the localized MMP and achieve magnetic field-assisted targeting for different clinical translations (78). Both designs provided desirable doxycycline release profiles (an initial burst followed by sustained release of 60 days) to inhibit the MMP activities and facilitate the preservation of the elastic matrix $(77,78)$. They also developed NPs for AAA tissue localized delivery of doxycycline, providing an active driving force for efficient uptake of intraluminally infused NPs to the AAA wall. Furthermore, they also rendered their polymer NPs mobile in an applied magnetic field via co-incorporation of superparamagnetics. The therapy can provide a non-surgical treatment option for high-risk AAA patients (78).

Statins were also loaded here in NP-assisted AAA treatment. Researchers developed a statin-loaded polymeric micelle to treat AAAs in rat models. The micelle showed medicinal efficacy by preventing aortic aneurysm expansion in a dosedependent manner. Furthermore, the micelle-injected group showed decreased macrophage infiltration and decreased MMP-9 activity in cases of AAA (79).

\section{Nanoparticles Targeting Inflammation and Vascular Smooth Muscle Cell Dysfunction}

NPs targeting AAA lesions can be used to treat developed AAA. Shirasu et al. reported an amphiphilic copolymer of poly(ethylene glycol)- $b$-poly ( $\gamma$-benzyl-L-glutamate) (PEG- $b$ PBLG) that was synthesized and formed a micellar NP via self-assembly (62). Shirasu et al. used this PEG- $b$-PBLG NP system for encapsulation and targeted delivery of rapamycin. It demonstrated a preferential uptake of NPs by macrophages at AAA lesions in vivo and enabled effective mitigation of aortic aneurysm expansion with a rapamycin dosage as low as $0.1 \mathrm{mg} / \mathrm{kg}$. In contrast, the free drug delivered at the same dosage failed to attenuate AAA (62). Later in 2020, Dhital et al. did further work to use a site-specific delivery system with targeted NPs to treat already developed AAA (72). They generated poly(ethylene glycol)-shelled NPs incorporating rapamycin, which has been evaluated for its potential in AAA treatment but caused many unwanted adverse effects in AAA 


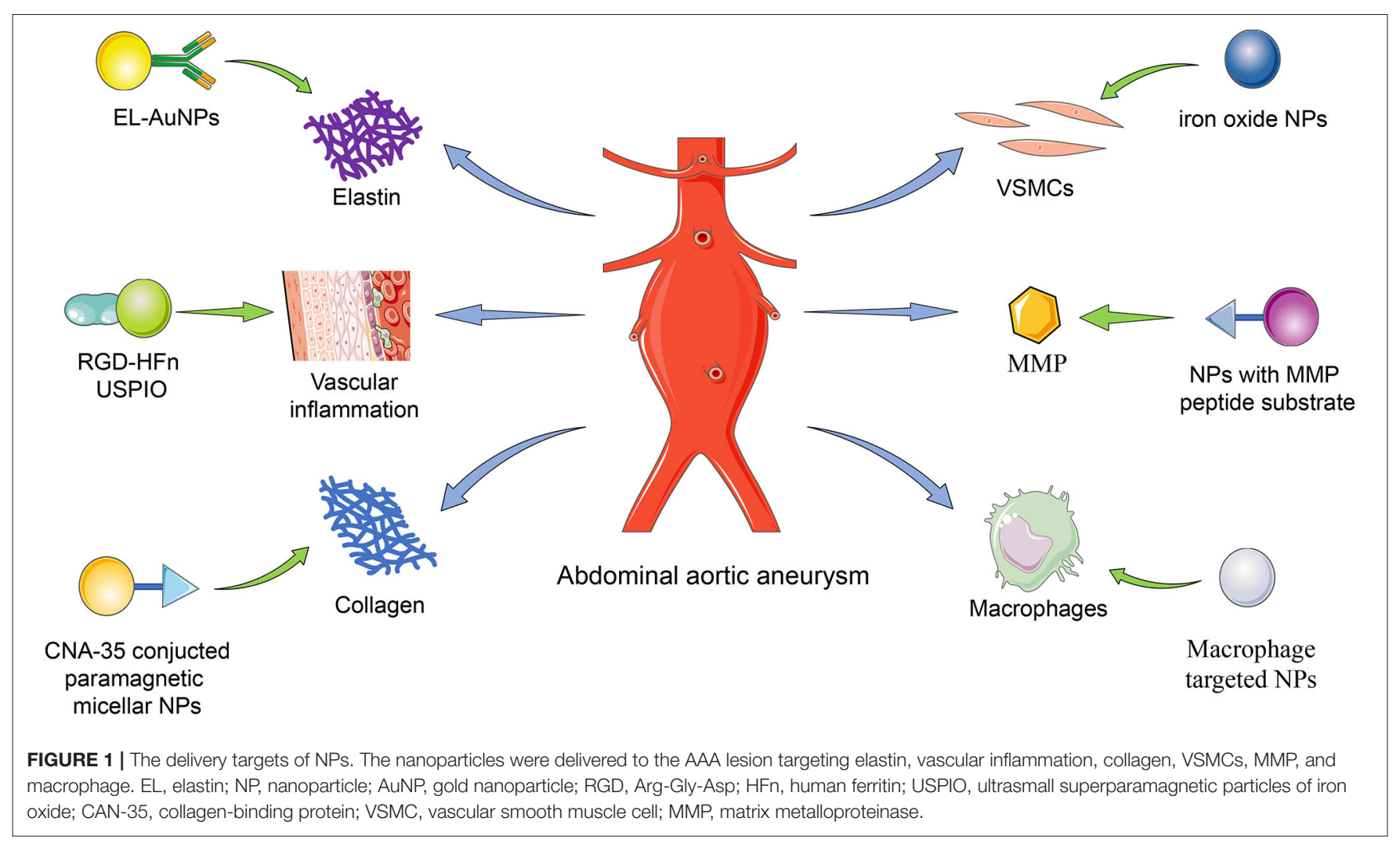

treatment when systematically used $(66,80)$. Rapamycin NPs injected during the AAA formation process evinced significant suppression of AAA formation and mural inflammation, providing specific rapamycin delivery to the rat AAA and contributing to establishing a drug therapy approach targeting aortic aneurysm lesions.

Cheng et al., on the other hand, capitalized on the high oxidative stress that typically occurs in AAA lesions. They developed reactive oxygen species (ROS)-responsive and targeted nanoplatforms to deliver rapamycin. In the in vitro experiments, this unique design proved responsive (e.g., rapamycin release) to the $\mathrm{H}_{2} \mathrm{O}_{2}$ condition and inhibitory to oxidative stress and VSMC apoptosis by acting as a ROS scavenger. When administered to a $\mathrm{CaCl}_{2}$-induced AAA rat model, this NP conjugated with $c$ (RGDfK) demonstrated the outstanding aneurysm-targeting ability. Furthermore, the rapamycin-loaded formulation exhibited clear therapeutic benefits, as indicated by reduced AAA size, MMP activity, macrophage infiltration, and endothelial deterioration (66).

Hyaluronic acid or hyaluronan (HA), a glycosaminoglycan (GAG) in the ECM (81), was reportedly able to stimulate elastin regeneration (82). Previous studies found that HA (HA-o) oligomers, especially the mixture of HA tetramers and hexamers, improved elastin assembly $(83,84)$. The negatively charged HAo may assist elastin fiber formation through interactions with cationic tropoelastin molecules and enhanced association with versican, a large chondroitin sulfate proteoglycan commonly found in the ECM (85). However, the administration of HA-o is challenging, most notably due to their susceptibility to proteolysis in the MMP-hyperactive aneurysmal environment $(65,86)$ and a short half-life in the bloodstream (87). The Ramamurthi group used PLGA NPs to protect the loaded HA-o. The VSMCs treated with HA-o-NP displayed enhanced deposition of elastin matrix and increased activity of lysyl oxidase (LOX), an enzyme essential for the cross-linking of elastin fibers (88). Moreover, MMP9 levels were reduced compared with the control of empty NP (no HA-o), whereas, MMP-2 levels were not significantly changed (67).

Transforming growth factor-beta 1 (TGF- $\beta 1$ ), a polypeptide that regulates cell growth, differentiation, proliferation, and apoptosis (89), is also an established elastogenic factor $(82,90)$. TGF- $\beta 1$ at higher concentrations can paradoxically induce the differentiation of SMCs to switch to an osteogenic phenotype promoting calcification (71, 91). The Ramamurthi group explored utilizing PLGA NPs to deliver TGF- $\beta 1$ and doxycycline to human aortic SMCs cultured in a $3 \mathrm{D}$ matrix (71). The SMCs treated with TGF- $\beta 1$-loaded NPs, or doxycycline-loaded NPs, showed significantly increased elastin content and matrix assembly relative to that treated with empty NP. According to these results in vitro, the authors accumulated that NP-assisted delivery holds the promise for TGF- $\beta 1$-based therapy in AAA management (71). 
TABLE 2 | Nanoparticles in AAA treatment.

\begin{tabular}{|c|c|c|c|c|c|c|}
\hline Nanoparticles & Targets & Component & Drugs loaded & Model & Administration & Detailed application \\
\hline EL-PEG-PLA & Elastase laminae & $\begin{array}{l}\text { Elastin antibody modified } \\
\text { PEGylated poly }\end{array}$ & BB-94 & $\begin{array}{l}\mathrm{CaCl} 2 \text {-induced rats, } \\
\text { Systemic calcifaction rats, } \\
\text { aherosclerotic plaque } \\
\text { created in } \mathrm{ApoE}^{-/-} \text {mice }\end{array}$ & Injection once & $\begin{array}{l}\text { Targeted delivery of } \\
\text { BB-94 loaded } \\
\text { EL-PEG-PLA NPs (8). }\end{array}$ \\
\hline PLGA NPS & MMP & $\begin{array}{l}\text { Coencapsulate with } \\
\text { doxycycline and SPION, } \\
\text { and } \\
\text { Cationic-functionalized } \\
\text { PLGA NPs. }\end{array}$ & $\begin{array}{l}\text { Doxycycline, } \\
\text { Statins }\end{array}$ & $\begin{array}{l}\text { Angll-infusion mice, } \\
\text { C57BL/6 mice underwent } \\
\text { transient elastase } \\
\text { perfusion }\end{array}$ & $\begin{array}{l}\text { Implanted once; oral } \\
\text { daily; }\end{array}$ & $\begin{array}{l}\text { Inhibit MMP enzymatic } \\
\text { function }(62) \text { and activities, } \\
\text { and facilitate the } \\
\text { preservation of the elastic } \\
\text { matrix }(63,64) \text {. }\end{array}$ \\
\hline HA-o-NP & VSMCs & HA loaded PLGA & $\mathrm{HA}$ & $\mathrm{CaCl} 2$-induced mice & $\begin{array}{l}\text { adenoviral gene } \\
\text { transfer }\end{array}$ & $\begin{array}{l}\text { Enhanced deposition of } \\
\text { elastin matrix and } \\
\text { increased LOX activity } \\
\text { (65). }\end{array}$ \\
\hline Drug -loaded NPs & SMCs & $\begin{array}{l}\text { TGF- } \beta 1 \text {-loaded NPs, } \\
\text { Doxycycline-loaded NPs }\end{array}$ & $\begin{array}{l}\text { TGF- } \beta 1 \text {, } \\
\text { Doxycycline }\end{array}$ & In vitro & & $\begin{array}{l}\text { Increased elastin content } \\
\text { and matrix assembly (66). }\end{array}$ \\
\hline PEG-b-PBLG & AAA lesion & Alexa647-labeled NPs & Rapamycin & $\begin{array}{l}\text { Porcine pancreatic } \\
\text { elastase mice }\end{array}$ & $\begin{array}{l}\text { IV route at 14-day and } \\
21 \text {-day }\end{array}$ & $\begin{array}{l}\text { Treating already } \\
\text { developed AAA (67). }\end{array}$ \\
\hline $\begin{array}{l}\text { NP-incorporating } \\
\text { rapamycin }\end{array}$ & AAA lesion & $\begin{array}{l}\text { Poly(ethyleneglycol)- } \\
\text { shelled nanoparticles } \\
\text { incorporating rapamycin }\end{array}$ & Rapamycin & $\begin{array}{l}\text { Porcine pancreatic } \\
\text { elastase mice }\end{array}$ & $\begin{array}{l}\text { IV route at 14-day and } \\
21 \text {-day }\end{array}$ & $\begin{array}{l}\text { Treat already developed } \\
\text { AAA (67). }\end{array}$ \\
\hline ROS-NPS & AAA lesion & $\begin{array}{l}\text { PEG-phospholipid, } \\
\text { oxidation-responsive } \\
\beta \text {-cyclodextrin derivative. }\end{array}$ & Rapamycin & $\begin{array}{l}\text { Porcine pancreatic } \\
\text { elastase rats }\end{array}$ & IV route at 7-day & $\begin{array}{l}\text { ROS-NPS can trigger the } \\
\text { release of payload to } \\
\text { control inflammation and } \\
\text { aneurysm growth (68). }\end{array}$ \\
\hline
\end{tabular}

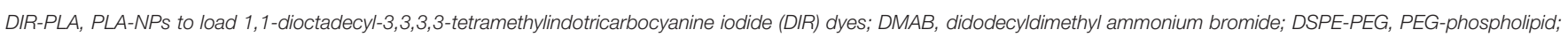

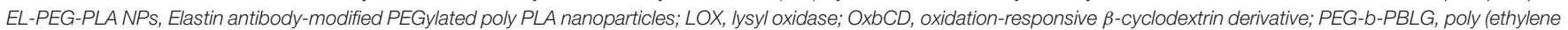

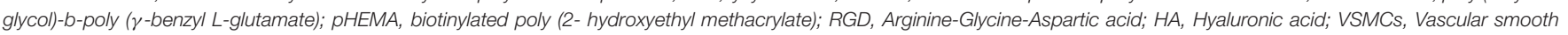
muscle cells.

\section{NANOPARTICLES IN ABDOMINAL AORTIC ANEURYSM STENT GRAFT}

In addition to targeting the vascular wall, some researchers focused on the stent graft targeted delivery system. A recent publication showed a novel drug delivery system, which allowed repetitively charging a graft with therapeutic drugs and releasing them to the aortic wall in vivo. The system was composed of a targeted graft labeled with a small target molecule and the target-recognizing nanocarrier, which contained suitable drugs. The drug released from this drug delivery system reduced the expression of MMP-9 in mouse aortas (74). This concept would allow systemic injection of drug-loaded NPs, thus enabling a more flexible therapeutic regimen. Under this scheme, a liposome-based biotinylated NP was developed that would specifically interact with the stent graft, which is coated with biotinylated poly(2-hydroxyethyl methacrylate) (pHEMA) (74). The avidin-biotin bond has long been known to be a robust non-covalent interaction (92). In this particular application, the pHEMA stent grafts were implanted in mice to mimic postEVAR AAA patients (74). By comparing the outcomes between the biotinylated and non-biotinylated NPs, either with or without drug encapsulation, this study demonstrated the targetability and feasibility for controlled drug release at AAA lesion site following systemic NP injection. With the ever-growing popularity of stent grafts and the persistent need for post-EVAR control of AAA progression, this particular study provides a unique concept for the future development of targeted nanoplatforms for both therapeutic and prognostic purposes.

Now, some researchers focused on the biodegradable nanofiber-loaded stent. A study in vitro demonstrated that the biodegradable nanofibers and the nanofiber-loaded stent graft provided sustained release of high vancomycin concentrations for up to 30 days. The in vivo study showed that the nanofiberloaded stent exhibited excellent biocompatibility and released high vancomycin concentrations into the local aortic wall for 8 weeks (93).

Another study elevated the long-term outcomes of implants in small animal models. In previous research, $\mathrm{Li}$ et al. fabricated a poly( $\varepsilon$-caprolactone) (PCL) bi-layered vascular graft consisting of an internal layer with circumferentially aligned microfibers and an external layer with random nanofibers. The circumferentially oriented VSMCs were successfully regenerated after the grafts were implanted in the rat abdominal aorta for 3 months. Then they investigated the long-term (18 months) performance of the bi-layered grafts in the same model. They found that most of the grafts' luminal surface circumferentially oriented VSMCs migrated to the grafts' luminal surface to form a neointima with uniform thickness. Accordingly, ECM including collagen, elastin, and GAG displayed high density in the neointima layer while low density in the walls of the graft because of the incomplete degradation of PCL. However, the contraction and relaxation function of regenerated neoartery almost disappeared. Other factors of grafts should be considered 
to achieve the regenerated artery similar to the native vessels after long-term implantation, but this is still a potential orientation in AAA graft (94). What is more, combining electrospinning and $3 \mathrm{D}$ printing to create patient-specific nanofiber tissueengineered vascular grafts (TEVGs) is a feasible technology as a future clinical option. Further, preclinical studies involving more complex anatomical shapes are warranted (24).

\section{DISCUSSION}

There have been numerous studies investigating the value of NPs in AAA imaging, treatment, and stent graft. However, although, NPs have shown high efficiency and low toxicity in these studies, there are still challenges for further clinical application. First, several animal models have been developed, such as the ANG II-induced AAA in ApoE-deficient mouse model, $\mathrm{CaCl}_{2}$-induced AAA model, and elastase-induced AAA model, but none of these models can accurately reproduce the pathogenic environment and disease progression of AAA patients. For instance, the complex hemodynamic changes in AAA are hard to achieve in these models. Second, the extended timing and costs, as well as potential immunogenic response, are also significant challenges to translate preclinical findings from animal models to human settings. Third, although, NPs conjugated with antibodies (e.g., EL-AuNPs) have high selectivity of AAA lesions, they are unstable in the organic solvent and are easy to denature during the conjugation process $(95,96)$. Compared with that, NPs conjugated with short peptides have better resistance to denaturation and have attracted more and more attention in exploring curative therapies of AAA (97).

In most of the current studies, NP-assisted imaging for AAA is based on a single imaging method. The combination of multiple imaging methods can be beneficial for improving the accuracy of AAA imaging. Combined ${ }^{18}$ F-FDG PET-CT and USPIO-MRI were used in a previous study for assessing tissue inflammation in AAA. ${ }^{18}$ F-FDG signal was focused mainly in the shoulder region of AAA, whereas, USPIO uptake was shown to be more apparent in the AAA body (29). In another study, combination of USPIOassisted MRI and computed tomography angiogram (CTA) was used for exploring the biological and mechanical properties of AAA. The results indicated that cellular inflammation and stress may represent different but complimentary aspects of AAA disease progression (56). A combined imaging approach with bioluminescence imaging (BLI) and MRI was used to study macrophage homing and accumulation in the experimental AAA disease (32). Similar dual imaging approaches may contribute to the study of AAA biology and the evaluation of novel therapies.

\section{REFERENCES}

1. Stather PW, Sidloff DA, Rhema IA, Choke E, Bown MJ, Sayers RD. A review of current reporting of abdominal aortic aneurysm mortality and prevalence in the literature. Eur J Vasc Endovasc Surg. (2014) 47:2402. doi: 10.1016/j.ejvs.2013.11.007

2. Sakalihasan N, Michel J, Katsargyris A, Kuivaniemi H, Defraigne J, Nchimi A, et al. Abdominal aortic aneurysms.
Therefore, future investigations should focus on the combination of several NP-assisted imaging.

The hemodynamics in the diseased abdominal aorta are considered to be a key contributor to the formation and growth of intraluminal thrombus (ILT) in AAA. In order to better understand the basic hemodynamic features in AAA imaging and seek potential treatment, many investigations have been made (98-102). Juchems et al. used computational fluid dynamics (CFD)-based blood flow simulation to analyze dynamic pressure in AAA before and after endovascular repair and provided a prognostic statement as to the possible homogenization of the pressure in abdominal stent grafts (98). Multidetector CT angiography (MDCT-A) and CFD were used to quantitatively and qualitatively assess the hemodynamic changes in AAA after stent-graft placement in another study (99). A threedimensional flow visualization method was proposed by Chen et al. to calculate wall stress in AAA patients (100). Quantitative understanding of NPs transport and adhesion dynamic is also very challenging due to complexity of fluid dynamics, and many attempts have been made in this field (103-105). In a recent study, USPIO uptake was used to assess the wall stress in AAA. Only $16 \%$ of aneurysms exhibited colocalization of elevated stress and USPIO uptake enhancement, suggesting that cellular inflammation and stress may represent different aspects of AAA disease progression (56).

In further research, more drug-coated NP delivery systems will be developed to get an AAA-targeted local treatment. Moreover, NPs in stent graft coated with therapeutic agents or targeted components have a promising prospect in AAA treatment.

\section{AUTHOR CONTRIBUTIONS}

$\mathrm{LY}$ and $\mathrm{KZ}$ wrote this manuscript. $\mathrm{ZL}$ provided the topic of this review and performed the critical revision of the manuscript. ZL and YS contributed to the concept of this manuscript. All authors read and approved the final manuscript.

\section{FUNDING}

This work was supported by the National Natural Science Foundation of China (81670433 and 81970398), the Project of Zhejiang Medical Young Talents (2017), Zhejiang Medical and Health Science and Technology Project (2020RC014), and the Natural Science Foundation of Zhejiang Province (Q20H020059). 
5. Lindeman JH, Matsumura JS. Pharmacologic management of aneurysms. Circ Res. (2019) 124:631-46. doi: 10.1161/CIRCRESAHA.118.312439

6. Prall AK, Longo GM, Mayhan WG, Waltke EA, Fleckten B, Thompson RW, et al. Doxycycline in patients with abdominal aortic aneurysms and in mice: comparison of serum levels and effect on aneurysm growth in mice. J Vasc Surg. (2002) 35:923-9. doi: 10.1067/mva.2002.123757

7. Ding R, McGuinness CL, Burnand KG, Sullivan E, Smith A. Matrix metalloproteinases in the aneurysm wall of patients treated with low-dose doxycycline. Vascular. (2005) 13:290-7. doi: 10.1258/rsmvasc.13.5.290

8. Zhu S, Zhu K, Li J, Lai H, Wang C. Nano-Biomaterials for the delivery of therapeutic and monitoring cues for aortic diseases. Front Bioeng Biotechnol. (2020) 8:583879. doi: 10.3389/fbioe.2020.583879

9. Sinha A, Shaporev A, Nosoudi N, Lei Y, Vertegel A, Lessner S, et al. Nanoparticle targeting to diseased vasculature for imaging and therapy. Nanomedicine. (2014) 10:1003-12. doi: 10.1016/j.nano.2014.02.002

10. Yodsanit N, Wang B, Zhao Y, Guo L, Kent K, Gong S. Recent progress on nanoparticles for targeted aneurysm treatment and imaging. Biomaterials. (2021) 265:120406. doi: 10.1016/j.biomaterials.2020.120406

11. Shamloo A, Ebrahimi S, Amani A, Fallah F. Targeted drug delivery of microbubble to arrest abdominal aortic aneurysm development: a simulation study towards optimized microbubble design. Sci Rep. (2020) 10:5393. doi: 10.1038/s41598-020-62410-3

12. Lim G. Nanotherapy for abdominal aortic aneurysm. Nat Rev Cardiol. (2019) 16:71. doi: 10.1038/s41569-018-0138-1

13. Aggarwal S, Qamar A, Sharma V, Sharma A. Abdominal aortic aneurysm: a comprehensive review. Exp Clin Cardiol. (2011) 16:11-5.

14. Emeto TI, Alele FO, Smith AM, Smith FM, Dougan T, Golledge J. Use of nanoparticles as contrast agents for the functional and molecular imaging of abdominal aortic aneurysm. Front Cardiovasc Med. (2017) 4:16. doi: $10.3389 /$ fcvm. 2017.00016

15. Pratt EC, Shaffer TM, Grimm J. Nanoparticles and radiotracers: advances toward radionanomedicine. Wiley Interdiscip Rev Nanomed Nanobiotechnol. (2016) 8:872-90. doi: 10.1002/wnan.1402

16. Shilo M, Reuveni T, Motiei M, Popovtzer R. Nanoparticles as computed tomography contrast agents: current status and future perspectives. Nanomedicine. (2012) 7:257-69. doi: 10.2217/nnm.11.190

17. Dobrucki LW, Sinusas AJ. Targeted imaging of abdominal aortic aneurysm: biology over structure. Circ Cardiovasc Imaging. (2020) 13:e010495. doi: 10.1161/CIRCIMAGING.120.010495

18. Toczek J, Meadows JL, Sadeghi MM. Novel molecular imaging approaches to abdominal aortic aneurysm risk stratification. Circ Cardiovasc Imaging. (2016) 9:e003023. doi: 10.1161/CIRCIMAGING.115.003023

19. Cole LE, Ross RD, Tilley JM, Vargo-Gogola T, Roeder RK. Gold nanoparticles as contrast agents in $\mathrm{x}$-ray imaging and computed tomography. Nanomedicine. (2015) 10:321-41. doi: 10.2217/nnm.14.171

20. Xi D, Dong S, Meng XX, Lu QH, Meng LJ, Ye J. Gold nanoparticles as computerized tomography (CT) contrast agents. RSC Adv. (2012) 2:1251524. doi: 10.1039/C2RA21263C

21. Meir R, Popovtzer R. Cell tracking using gold nanoparticles and computed tomography imaging. Wiley Interdiscip Rev Nanomed Nanobiotechnol. (2018) 10:1-15. doi: 10.1002/wnan.1480

22. Stockhofe K, Postema JM, Schieferstein H, Ross TL. Radiolabeling of nanoparticles and polymers for PET imaging. Pharmaceuticals. (2014) 7:392418. doi: 10.3390/ph7040392

23. Evelhoch JL. In vivo MR in the drug pipeline. J Magn Reson. (2018) 292:11728. doi: 10.1016/j.jmr.2018.04.012

24. Golestani R, Sadeghi MM. Emergence of molecular imaging of aortic aneurysm: implications for risk stratification and management. $\mathrm{J} \mathrm{Nucl}$ Cardiol. (2014) 21:251-67; quiz 68-70. doi: 10.1007/s12350-013-9845-5

25. Dale MA, Ruhlman MK, Baxter BT. Inflammatory cell phenotypes in AAAs: their role and potential as targets for therapy. Arterioscler Thromb Vasc Biol. (2015) 35:1746-55. doi: 10.1161/ATVBAHA.115.305269

26. Kitagawa T, Kosuge H, Uchida M, Iida Y, Dalman RL, Douglas T, et al. RGD targeting of human ferritin iron oxide nanoparticles enhances in vivo MRI of vascular inflammation and angiogenesis in experimental carotid disease and abdominal aortic aneurysm. J Magn Reson Imaging. (2017) 45:1144-53. doi: 10.1002/jmri.25459
27. Richards JM, Semple SI, MacGillivray TJ, Gray C, Langrish JP, Williams M, et al. Abdominal aortic aneurysm growth predicted by uptake of ultrasmall superparamagnetic particles of iron oxide: a pilot study. Circ Cardiovasc Imaging. (2011) 4:274-81. doi: 10.1161/CIRCIMAGING.110.959866

28. Heidt T, Nahrendorf M. Multimodal iron oxide nanoparticles for hybrid biomedical imaging. NMR Biomed. (2013) 26:75665. doi: $10.1002 / \mathrm{nbm} .2872$

29. McBride OM, Joshi NV, Robson JM, MacGillivray TJ, Gray CD, Fletcher $\mathrm{AM}$, et al. Positron emission tomography and magnetic resonance imaging of cellular inflammation in patients with abdominal aortic aneurysms. Eur J Vasc Endovasc Surg. (2016) 51:518-26. doi: 10.1016/j.ejvs.2015.12.018

30. Razavian M, Marfatia R, Mongue-Din H, Tavakoli S, Sinusas AJ, Zhang $J$, et al. Integrin-targeted imaging of inflammation in vascular remodeling. Arterioscler Thromb Vasc Biol. (2011) 31:2820-6. doi: 10.1161/ATVBAHA.111.231654

31. Yao Y, Wang $\mathrm{Y}$, Zhang $\mathrm{Y}, \mathrm{Li} \mathrm{Y}$, Sheng $\mathrm{Z}$, Wen $\mathrm{S}$, et al. In vivo imaging of macrophages during the early-stages of abdominal aortic aneurysm using high resolution MRI in ApoE mice. PLoS ONE. (2012) 7:e33523. doi: 10.1371/journal.pone.0033523

32. Miyama N, Dua MM, Schultz GM, Kosuge H, Terashima M, Pisani LJ, et al. Bioluminescence and magnetic resonance imaging of macrophage homing to experimental abdominal aortic aneurysms. Mol Imaging. (2012) 11:126-34. doi: 10.2310/7290.2011.00033

33. Kotze CW, Rudd JHF, Ganeshan B, Menezes LJ, Brookes J, Agu O, et al. CT signal heterogeneity of abdominal aortic aneurysm as a possible predictive biomarker for expansion. Atherosclerosis. (2014) 233:5107. doi: 10.1016/j.atherosclerosis.2014.01.001

34. Badawi MI. Effect of gold nanoparticles contrast agent concentration on x-ray diagnoses: experimental and computational study. (2014) 2:639. doi: 10.11648/j.nano.20140204.11

35. Au JT, Craig G, Longo V, Zanzonico P, Mason M, Fong Y, et al. Gold nanoparticles provide bright long-lasting vascular contrast for CT imaging. AJR Am J Roentgenol. (2013) 200:1347-51. doi: 10.2214/AJR.12. 8933

36. Wang X, Lane BA, Eberth JF, Lessner SM, Vyavahare NR. Gold nanoparticles that target degraded elastin improve imaging and rupture prediction in an AngII mediated mouse model of abdominal aortic aneurysm. Theranostics. (2019) 9:4156-67. doi: 10.7150/thno.34441

37. Lane BA, Wang X, Lessner SM, Vyavahare NR, Eberth JF. Targeted gold nanoparticles as an indicator of mechanical damage in an elastase model of aortic aneurysm. Ann Biomed Eng. (2020) 48:2268-78. doi: 10.1007/s10439-020-02500-5

38. Sakalihasan N, Van Damme H, Gomez P, Rigo P, Lapiere CM, Nusgens $\mathrm{B}$, et al. Positron emission tomography (PET) evaluation of abdominal aortic aneurysm (AAA). Eur J Vasc Endovasc Surg. (2002) 23:4316. doi: 10.1053/ejvs.2002.1646

39. Kotze CW, Groves AM, Menezes LJ, Harvey R, Endozo R, Kayani IA, et al. What is the relationship between 8F-FDG aortic aneurysm uptake on PET/CT and future growth rate? Eur J Nucl Med Mol Imaging. (2011) 38:1493-9. doi: 10.1007/s00259-011-1799-8

40. Marini C, Morbelli S, Armonino R, Spinella G, Riondato M, Massollo $M$, et al. Direct relationship between cell density and FDG uptake in asymptomatic aortic aneurysm close to surgical threshold: an in vivo and in vitro study. Eur J Nucl Med Mol Imaging. (2012) 39:91101. doi: 10.1007/s00259-011-1955-1

41. Morel O, Mandry D, Micard E, Kauffmann C, Lamiral Z, Verger A, et al. Evidence of cyclic changes in the metabolism of abdominal aortic aneurysms during growth phases: 8 F-FDG PET sequential observational study. J Nucl Med. (2015) 56:1030-5. doi: 10.2967/jnumed.114.146415

42. Truijers M, Kurvers HA, Bredie SJ, Oyen WJ, Blankensteijn JD. In vivo imaging of abdominal aortic aneurysms: increased FDG uptake suggests inflammation in the aneurysm wall. J Endovasc Ther. (2008) 15:4627. doi: 10.1583/08-2447.1

43. Palombo D, Morbelli S, Spinella G, Pane B, Marini C, Rousas N, et al. A positron emission tomography/computed tomography (PET/CT) evaluation of asymptomatic abdominal aortic aneurysms: another point of view. Ann Vasc Surg. (2012) 26:491-9. doi: 10.1016/j.avsg.2011.05.038 
44. Courtois A, Nusgens BV, Hustinx R, Namur G, Gomez P, Somja J, et al. 18FFDG uptake assessed by PET/CT in abdominal aortic aneurysms is associated with cellular and molecular alterations prefacing wall deterioration and rupture. J Nucl Med. (2013) 54:1740-7. doi: 10.2967/jnumed.112.115873

45. Tegler G, Ericson K, Sörensen J, Björck M, Wanhainen A. Inflammation in the walls of asymptomatic abdominal aortic aneurysms is not associated with increased metabolic activity detectable by 18fluorodeoxglucose positron-emission tomography. J Vasc Surg. (2012) 56:802-7. doi: 10.1016/j.jvs.2012.02.024

46. Barwick TD, Lyons OT, Mikhaeel NG, Waltham M, O'Doherty MJ. 18F-FDG PET-CT uptake is a feature of both normal diameter and aneurysmal aortic wall and is not related to aneurysm size. Eur J Nucl Med Mol Imaging. (2014) 41:2310-8. doi: 10.1007/s00259-014-2865-9

47. Jalalzadeh H, Indrakusuma R, Planken RN, Legemate DA, Koelemay MJ, Balm R. Inflammation as a predictor of abdominal aortic aneurysm growth and rupture: a systematic review of imaging biomarkers. Eur J Vasc Endovasc Surg. (2016) 52:333-42. doi: 10.1016/j.ejvs.2016.05.002

48. Polyak A, Ross TL. Nanoparticles for SPECT and pet imaging: towards personalized medicine and theranostics. Curr Med Chem. (2018) 25:432853. doi: 10.2174/0929867324666170830095553

49. Nahrendorf M, Keliher E, Marinelli B, Leuschner F, Robbins CS, Gerszten $\mathrm{RE}$, et al. Detection of macrophages in aortic aneurysms by nanoparticle positron emission tomography-computed tomography. Arterioscler Thromb Vasc Biol. (2011) 31:750-7. doi: 10.1161/ATVBAHA.110.221499

50. Richard C, Doan BT, Beloeil JC, Bessodes M, Tóth E, Scherman D. Noncovalent functionalization of carbon nanotubes with amphiphilic gd3+ chelates: toward powerful t 1 and t2 MRI contrast agents. Nano Lett. (2008) 8:232-6. doi: 10.1021/nl072509z

51. Deux JF, Dai J, Rivière C, Gazeau F, Méric P, Gillet B, et al. Aortic aneurysms in a rat model: in vivo MR imaging of endovascular cell therapy. Radiology. (2008) 246:185-92. doi: 10.1148/radiol.2461070032

52. Nordon IM, Hinchliffe RJ, Loftus IM, Thompson MM. Pathophysiology and epidemiology of abdominal aortic aneurysms. Nat Rev Cardiol. (2011) 8:92-102. doi: 10.1038/nrcardio.2010.180

53. Klink A, Heynens J, Herranz B, Lobatto ME, Arias T, Sanders HM, et al. In vivo characterization of a new abdominal aortic aneurysm mouse model with conventional and molecular magnetic resonance imaging. J Am Coll Cardiol. (2011) 58:2522-30. doi: 10.1016/j.jacc.2011.09.017

54. Raffort J, Lareyre F, Clément M, Hassen-Khodja R, Chinetti G, Mallat Z. Monocytes and macrophages in abdominal aortic aneurysm. Nat Rev Cardiol. (2017) 14:457-71. doi: 10.1038/nrcardio.2017.52

55. Sadat U, Taviani V, Patterson AJ, Young VE, Graves MJ, Teng Z, et al. Ultrasmall superparamagnetic iron oxide-enhanced magnetic resonance imaging of abdominal aortic aneurysms-a feasibility study. Eur J Vasc Endovasc Surg. (2011) 41:167-74. doi: 10.1016/j.ejvs.2010.08.022

56. Conlisk N, Forsythe RO, Hollis L, Doyle BJ, McBride OMB, Robson JMJ, et al. Exploring the biological and mechanical properties of abdominal aortic aneurysms using uspio mri and peak tissue stress: a combined clinical and finite element study. J Cardiovasc Transl Res. (2017) 10:48998. doi: 10.1007/s12265-017-9766-9

57. Ichihashi S, Marugami N, Tanaka T, Iwakoshi S, Kurumatani N, Kitano S, et al. Preliminary experience with superparamagnetic iron oxide-enhanced dynamic magnetic resonance imaging and comparison with contrastenhanced computed tomography in endoleak detection after endovascular aneurysm repair. J Vasc Surg. (2013) 58:66-72. doi: 10.1016/j.jvs.2012.12.061

58. Yao Y, Cheng K, Cheng Z. Evaluation of a smart activatable MRI nanoprobe to target matrix metalloproteinases in the earlystages of abdominal aortic aneurysms. Nanomedicine. (2020) 26:102177. doi: 10.1016/j.nano.2020.102177

59. Kitagawa T, Kosuge H, Uchida M, Dua MM, Iida Y, Dalman RL, et al. RGDconjugated human ferritin nanoparticles for imaging vascular inflammation and angiogenesis in experimental carotid and aortic disease. Mol Imaging Biol. (2012) 14:315-24. doi: 10.1007/s11307-011-0495-1

60. Knopp T, Gdaniec N, Möddel M. Magnetic particle imaging: from proof of principle to preclinical applications. Phys Med Biol. (2017) 62:R12478. doi: 10.1088/1361-6560/aa6c99

61. Mangarova DB, Brangsch J, Mohtashamdolatshahi A, Kosch O, Paysen $\mathrm{H}$, Wiekhorst $\mathrm{F}$, et al. Ex vivo magnetic particle imaging of vascular inflammation in abdominal aortic aneurysm in a murine model. Sci Rep. (2020) 10:12410. doi: 10.1038/s41598-020-69299-y

62. Shirasu T, Koyama H, Miura Y, Hoshina K, Kataoka K, Watanabe T. Nanoparticles effectively target rapamycin delivery to sites of experimental aortic aneurysm in rats. PLoS ONE. (2016) 11:e0157813. doi: 10.1371/journal.pone.0157813

63. Bartoli MA, Parodi FE, Chu J, Pagano MB, Mao D, Baxter BT, et al. Localized administration of doxycycline suppresses aortic dilatation in an experimental mouse model of abdominal aortic aneurysm. Ann Vasc Surg. (2006) 20:22836. doi: 10.1007/s10016-006-9017-z

64. Yamawaki-Ogata A, Hashizume R, Satake M, Kaneko H, Mizutani S, Moritan T, et al. A doxycycline loaded, controlled-release, biodegradable fiber for the treatment of aortic aneurysms. Biomaterials. (2010) 31:955464. doi: 10.1016/j.biomaterials.2010.08.069

65. Isnard N, Robert L, Renard G. Effect of sulfated GAGs on the expression and activation of MMP-2 and MMP-9 in corneal and dermal explant cultures. Cell Biol Int. (2003) 27:779-84. doi: 10.1016/S1065-6995(03)00167-7

66. Cheng J, Zhang R, Li C, Tao H, Dou Y, Wang Y, et al. A targeting nanotherapy for abdominal aortic aneurysms. J Am Coll Cardiol. (2018) 72:2591-605. doi: 10.1016/j.jacc.2018.08.2188

67. Sylvester A, Sivaraman B, Deb P, Ramamurthi A. Nanoparticles for localized delivery of hyaluronan oligomers towards regenerative repair of elastic matrix. Acta Biomater. (2013) 9:9292-302. doi: 10.1016/j.actbio.2013.07.032

68. Nosoudi N, Chowdhury A, Siclari S, Karamched S, Parasaram V, Parrish $\mathrm{J}$, et al. Reversal of vascular calcification and aneurysms in a rat model using dual targeted therapy with EDTA- and PGG-loaded nanoparticles. Theranostics. (2016) 6:1975-87. doi: 10.7150/thno.16547

69. Nosoudi N, Nahar-Gohad P, Sinha A, Chowdhury A, Gerard P, Carsten CG, et al. Prevention of abdominal aortic aneurysm progression by targeted inhibition of matrix metalloproteinase activity with batimastat-loaded nanoparticles. Circ Res. (2015) 117:e80-9. doi: 10.1161/CIRCRESAHA.115.307207

70. Nosoudi N, Chowdhury A, Siclari S, Parasaram V, Karamched S, Vyavahare N. Systemic delivery of nanoparticles loaded with pentagalloyl glucose protects elastic lamina and prevents abdominal aortic aneurysm in rats. $J$ Cardiovasc Transl Res. (2016) 9:445-55. doi: 10.1007/s12265-016-9709-x

71. Venkataraman L, Sivaraman B, Vaidya P, Ramamurthi A. Nanoparticulate delivery of agents for induced elastogenesis in three-dimensional collagenous matrices. J Tissue Eng Regen Med. (2016) 10:1041-56. doi: 10.1002/term.1889

72. Dhital S, Vyavahare NR. Nanoparticle-based targeted delivery of pentagalloyl glucose reverses elastase-induced abdominal aortic aneurysm and restores aorta to the healthy state in mice. PLoS ONE. (2020) 15:e0227165. doi: 10.1371/journal.pone.0227165

73. Camardo A, Carney S, Ramamurthi A. Assessing the targeting and fate of cathepsin $\mathrm{k}$ antibody-modified nanoparticles in a rat abdominal aortic aneurysm model. Acta Biomater. (2020) 112:225-33. doi: 10.1016/j.actbio.2020.05.037

74. Yoshimura K, Aoki H, Teruyama C, Iijima M, Tsutsumi H, Kuroda Si, et al. A novel hybrid drug delivery system for treatment of aortic aneurysms. Int J Mol Sci. (2020) 21:5538. doi: 10.3390/ijms21155538

75. Erba E, Ronzoni S, Bassano L, Giavazzi R, D’Incalci M. The metalloproteinase inhibitor batimastat (BB-94) causes cell cycle phase perturbations in ovarian cancer cells. Ann Oncol. (1999) 10:589-91. doi: 10.1023/A:1026438801080

76. Sivaraman B, Ramamurthi A. Multifunctional nanoparticles for doxycycline delivery towards localized elastic matrix stabilization and regenerative repair. Acta Biomater. (2013) 9:6511-25. doi: 10.1016/j.actbio.2013. 01.023

77. Camardo A, Seshadri D, Broekelmann T, Mecham R, Ramamurthi A. Multifunctional, JNK-inhibiting nanotherapeutics for augmented elastic matrix regenerative repair in aortic aneurysms. Drug Deliv Transl Res. (2018) 8:964-84. doi: 10.1007/s13346-017-0419-y

78. Sivaraman B, Swaminathan G, Moore L, Fox J, Seshadri D, Dahal S, et al. Magnetically-responsive, multifunctional drug delivery nanoparticles for elastic matrix regenerative repair. Acta Biomater. (2017) 52:17186. doi: 10.1016/j.actbio.2016.11.048

79. Fukuhara N, Honda Y, Ukita N, Matsui M, Miura Y, Hoshina K. Efficient suppression of abdominal aortic aneurysm expansion in rats through 
systemic administration of statin-loaded nanomedicine. Int J Mol Sci. (2020) 21:8702. doi: 10.3390/ijms21228702

80. Dou Y, Chen Y, Zhang X, Xu X, Chen Y, Guo J, et al. Non-proinflammatory and responsive nanoplatforms for targeted treatment of atherosclerosis. Biomaterials. (2017) 143:93-108. doi: 10.1016/j.biomaterials.2017.07.035

81. Al-Ghananeem AM, Malkawi AH, Muammer YM, Balko JM, Black EP, Mourad W, et al. Intratumoral delivery of Paclitaxel in solid tumor from biodegradable hyaluronan nanoparticle formulations. AAPS PharmSciTech. (2009) 10:410-7. doi: 10.1208/s12249-009-9222-5

82. Gacchina CE, Deb P, Barth JL, Ramamurthi A. Elastogenic inductability of smooth muscle cells from a rat model of late stage abdominal aortic aneurysms. Tissue Eng Part A. (2011) 17:1699-711. doi: 10.1089/ten.tea.2010.0526

83. Joddar B, Ramamurthi A. Fragment size- and dose-specific effects of hyaluronan on matrix synthesis by vascular smooth muscle cells. Biomaterials. (2006) 27:2994-3004. doi: 10.1016/j.biomaterials.2006.01.020

84. Joddar B, Ramamurthi A. Elastogenic effects of exogenous hyaluronan oligosaccharides on vascular smooth muscle cells. Biomaterials. (2006) 27:5698-707. doi: 10.1016/j.biomaterials.2006.07.020

85. Wight TN. Versican: a versatile extracellular matrix proteoglycan in cell biology. Curr Opin Cell Biol. (2002) 14:61723. doi: 10.1016/S0955-0674(02)00375-7

86. Isnard N, Legeais JM, Renard G, Robert L. Effect of hyaluronan on MMP expression and activation. Cell Biol Int. (2001) 25:7359. doi: 10.1006/cbir.2001.0759

87. Fraser JR, Laurent TC, Engström-Laurent A, Laurent UG. Elimination of hyaluronic acid from the blood stream in the human. Clin Exp Pharmacol Physiol. (1984) 11:17-25. doi: 10.1111/j.1440-1681.1984.tb00235.x

88. Onoda M, Yoshimura K, Aoki H, Ikeda Y, Morikage N, Furutani A, et al. Lysyl oxidase resolves inflammation by reducing monocyte chemoattractant protein-1 in abdominal aortic aneurysm. Atherosclerosis. (2010) 208:3669. doi: 10.1016/j.atherosclerosis.2009.07.036

89. Ghadami M, Makita Y, Yoshida K, Nishimura G, Fukushima Y, Wakui $\mathrm{K}$, et al. Genetic mapping of the Camurati-Engelmann disease locus to chromosome 19q13.1-q13.3. Am J Hum Genet. (2000) 66:1437. doi: $10.1086 / 302728$

90. Gacchina C, Brothers T, Ramamurthi A. Evaluating smooth muscle cells from $\mathrm{CaCl} 2$-induced rat aortal expansions as a surrogate culture model for study of elastogenic induction of human aneurysmal cells. Tissue Eng Part A. (2011) 17:1945-58. doi: 10.1089/ten.tea.2010.0475

91. Simionescu A, Philips K, Vyavahare N. Elastin-derived peptides and TGFbetal induce osteogenic responses in smooth muscle cells. Biochem Biophys Res Commun. (2005) 334:524-32. doi: 10.1016/j.bbrc.2005.06.119

92. Jain A, Cheng K. The principles and applications of avidin-based nanoparticles in drug delivery and diagnosis. J Control Release. (2017) 245:27-40. doi: 10.1016/j.jconrel.2016.11.016

93. Liu K-S, Lee C-H, Lee D, Liu M, Tsai F-C, Tseng Y-Y. Sustained local delivery of high-concentration vancomycin from a hybrid biodegradable, antibiotic-eluting, nanofiber-loaded endovascular prosthesis for treatment of mycotic aortic aneurysms. J Vasc Surg. (2018) 68:597-606. doi: 10.1016/j.jvs.2017.07.142

94. Li W, Chen J, Xu P, Zhu M, Wu Y, Wang Z, et al. Long-term evaluation of vascular grafts with circumferentially aligned microfibers in a rabdominal aorta replacement model. J Biomed Mater Res B Appl Biomater. (2018) 106:2596-604. doi: 10.1002/jbm.b.34076
95. Rehan M, Younus H. Effect of organic solvents on the conformation and interaction of catalase and anticatalase antibodies. Int J Biol Macromol. (2006) 38:289-95. doi: 10.1016/j.ijbiomac.2006.03.023

96. Wang W, Singh S, Zeng DL, King K, Nema S. Antibody structure, instability, and formulation. J Pharm Sci. (2007) 96:1-26. doi: 10.1002/jps.20727

97. Schaffner P, Dard MM. Structure and function of RGD peptides involved in bone biology. Cell Mol Life Sci. (2003) 60:119-32. doi: 10.1007/s000180300008

98. Juchems MS, Pless D, Fleiter TR, Gabelmann A, Liewald F, Brambs HJ, et al. [Non-invasive, multi detector row (MDR) CT based computational fluid dynamics (CFD) analysis of hemodynamics in infrarenal abdominal aortic aneurysm (AAA) before and after endovascular repair]. Rofo. (2004) 176:56-61. doi: 10.1055/s-2004-812966

99. Frauenfelder T, Lotfey M, Boehm T, Wildermuth S. Computational fluid dynamics: hemodynamic changes in abdominal aortic aneurysm after stent-graft implantation. Cardiovasc Intervent Radiol. (2006) 29:61323. doi: 10.1007/s00270-005-0227-5

100. Chen CY, Antón R, Hung MY, Menon P, Finol EA, Pekkan K. Effects of intraluminal thrombus on patient-specific abdominal aortic aneurysm hemodynamics via stereoscopic particle image velocity and computational fluid dynamics modeling. J Biomech Eng. (2014) 136:031001. doi: 10.1115/1.4026160

101. Lu YH, Mani K, Panigrahi B, Hsu WT, Chen CY. Endoleak assessment using computational fluid dynamics and image processing methods in stented abdominal aortic aneurysm models. Comput Math Methods Med. (2016) 2016:9567294. doi: 10.1155/2016/9567294

102. Dubey A, Vasu B, Anwar Bég O, Gorla RSR, Kadir A. Computational fluid dynamic simulation of two-fluid non-Newtonian nanohemodynamics through a diseased artery with a stenosis and aneurysm. Comput Methods Biomech Biomed Engin. (2020) 23:345-71. doi: 10.1080/10255842.2020.1729755

103. Sohrabi S, Yunus DE, Xu J, Yang J, Liu Y. Characterization of nanoparticle binding dynamics in microcirculation using an adhesion probability function. Microvasc Res. (2016) 108:41-7. doi: 10.1016/j.mvr.2016. 07.005

104. Kolitsi LI, Yiantsios SG. Transport of nanoparticles in magnetic targeting: comparison of magnetic, diffusive and convective forces and fluxes in the microvasculature, through vascular pores and across the interstitium. Microvasc Res. (2020) 130:104007. doi: 10.1016/j.mvr.2020.10 4007

105. Tripathi D, Prakash J, Tiwari AK, Ellahi R. Thermal, microrotation, electromagnetic field and nanoparticle shape effects on $\mathrm{Cu}$ $\mathrm{CuO} /$ blood flow in microvascular vessels. Microvasc Res. (2020) 132:104065. doi: 10.1016/j.mvr.2020.104065

Conflict of Interest: The authors declare that the research was conducted in the absence of any commercial or financial relationships that could be construed as a potential conflict of interest.

Copyright (C) 2021 Yin, Zhang, Sun and Liu. This is an open-access article distributed under the terms of the Creative Commons Attribution License (CC BY). The use, distribution or reproduction in other forums is permitted, provided the original author(s) and the copyright owner(s) are credited and that the original publication in this journal is cited, in accordance with accepted academic practice. No use, distribution or reproduction is permitted which does not comply with these terms. 